\title{
Review of: "Catalytic asymmetric nucleophilic fluorination using BF3·Et2O as fluorine source and activating reagent"
}

Pinhong Chen

Potential competing interests: The author(s) declared that no potential competing interests exist.

I like the words "a small atom with a big ego". Indeed, fluorine is important in the fields of organic chemistry, pharmaceuticals, agrochemicals and material chemistry. And the asymmetric fluorinations with fluoride as cheap fluorine source make the fluorine chemistry applicable in pharmaceuticals. This paper published by Prof. Xianxing Jiang disclosed a catalytic asymmetric nucleophilic fluorination using $\mathrm{BF}_{3} \cdot \mathrm{Et}_{2} \mathrm{O}$ as the fluorine reagent in the presence of chiral iodine catalyst. Chiral iodine catalysis has been applied in asymmetric oxygenation and amination reactions, and Prof. Nevado only achieved moderate enantioselectivity in fluorinations. Thus, this is not totally new chemistry, but the paper showed that various chiral fluorinated oxazine products were obtained with good to excellent enantioselectivities (up to $>99 \%$ ee) and diastereoselectivities (up to $>20: 1 \mathrm{dr}$ ). I give a good rate for this paper, because it is a synthetic uesful chemistry. 